\title{
TRANSCRIÇÃO PORTUGUESA DE UM FONEMA TUPI
}

\section{R. F. MANSUR GUERIOS}

Por que as palavras portuguesas de origem tupi, nas quais há - fonema s, são grafadas com $c_{\text {, }}$ ç e não com s, ss? Sejam exemplos Ceará, Ceraíma, Cemoaba, cipo, ciricica, igaçaba, piracema, jacina, paçoca, muriçoca, Iguaçu. Será que foi tão-só por uma convenção, como, p. ex., sapato, em vez de çapato, sujo, em lugar de çujo?

Os vocábulos tomados de empréstimo a qualquer língua, se têm os "mesmos" fonemas do idioma que os acolheu, são reproduzidos ossim, "grosso modo", sem modificação.

E quando os fonemas são desconhecidos, vêm a ser reproduzidos com adaptação: Acham-se neste caso os exemplares: órabe atfahona $>$ port. atafone, alemão Kuchen $>$ por. cuque, italiano ciarlare $>$ port. charlar, francês purée $>$ port. purê, pirê, grego pháselos $>$ faselus, ao lado de phaselus, grego chártes $>$ lat. carta, do lado de charta, grego theios > lat. lius, ao lado de thius, etc.

Ora, quando o tupi do litoral, tupi antigo, foi transcrito pelos primeiros missionários e pelos demais que o estudaram, fizeram-no com relativa perfeição. O Pe. José de Anchieta, p. ex., na sua ARTE DE GRAMATICA DA LINGUA MAIS USADA NA COSTA DO BRASIL ", (1995), transcreve com $\mathrm{c}$ e $\mathrm{c}$ em vez de s (surdo): "Em lugar do s. in principio, ou medio dictionis serue, $\boldsymbol{s}$, com zeura, vt Açô, çată" (cap. I, p. 1). E medial: ndaçói, çarã, çára, teçá, xèci, etc. ("passim"). No cap. II, p. 5, esta nota: "Ce, $\mathrm{Ci}$, hão se de pronunciar, como que tivessem zeura como no Portugues, cera, cidra, vt acêm, acic". Em outras obras do Pe. Anchieta observa-se a mesma transcrição. P. ex., n' O AUTO DE SAO LOURENÇO, peça, teatral: çeră, caugoaçu, Araçatiba, etc. ${ }^{2}$ Das POESIAS TUPIS (séc. 16) atribuídas ao citado cateÇuista: porace:, ayucei, cerapoá, cic, mboeçara, etc. ${ }^{3}$

1 ANCHIETA. José de. Arte de gramática da linga maik usada na costa do Branil. Ed. facsimilar. Sāo Paulo, Ed. Archieta, 1946. 68 f.

2 BARbosa. A. Lemos. O Auto de São Lourenço; uma peça teatral de Anchieta em tuni, castelhano e portuguề. Verbam, $7(2): 201-17$, jun. 1950.

8 MARTins, M. L. P. Poesins tupis (séc. XVI). Boletim da Faculdade de Fllosofia, Ciencias - Letres de USP n. ${ }^{\circ} 51,1946$. 
De um artigo do Pe. Serafim Leite, S.J. com o título LEONARDO DO VALE, AUTOR DO PRIMEIRO "VOCABULARIO NA LINGUA BRASÍlCA" (1591) extraio, entre outros, estes exemplos que aquele historiador menciona: Jupuguàçuqueçaba, igçauba, çupia, itaciba, uça, ciri ${ }^{4}$.

No CATECISMO NA LíngUA BRASILICA do Pe. Antonio de Araújo (reprodução fac-similar da $1 .^{\circ}$ edição, 1618) há símiles exemplos: xepoçang, recê, çaauçubi pira çoçê, etc. ${ }^{5}$

No VOCABULARIO DA LINGUA BRASILICA, ano de 1621 são encontradiços çupe, abarêguaçú, teçâ, coece, etc. ${ }^{6}$

Na GRAMATICA DA LINGUA DO BRASIL, facsímile da edição de 1687, do Pe. Luís Figueira: "Fição excluidas, F, L, S, Z" (p. 1), e na p. 2 as transcrições aiucéi, "desejo comer", açepùi, "borrifo" (v.), "borrifo" (v.), açaçub, "amo" (v.), çapyu, "ele abrasa", etc. 7

N'O CADERNO DA LINGUA OU VOCABULARIO PORTUGUES TUPI de Frei João de Arronches (manuscrito de 1739), dado a luz em 1795: truruçu, teçá, coecé, çobá, çupi, cetá, etc. ${ }^{8}$

NO DICIONARIO PORTUGUES - BRASILIANO E BRASILIANO PORTUGUES, "reimpressão integral da edição de 1795, seguida da 2. ${ }^{\circ}$ parte até hoje inédita, ordenada e prefaciada por Plínio $M$. da Sılva Ayrcsa": coaracy, turuçu, çobâ, ceçâ, etc ${ }^{9}$

No VOCABULÁRIO PORTUGUES - BRASILICO ("copiado em Piratininga em $1621^{\prime \prime}$ : çupe, ereçó, guáçu, çai, cebaê, açoiâbs, etc.,

4 LEITE, Serafim. Leonardo do Vale, autor do primeiro "Vocabulário na Ifngae bresflica". Verbum, 1(1):18, 1944.

5 ARAUJO, Antonio. Catecismo na língua brasílica. Reprod. facsiliar de 1 ed., 1618. Rio deJaneiro. Pontificia Universidade Católica, 1952. 179 l.

6 Vocabulario na lingua brasílica. 2.* ed. rev. por Carlos Drumond. Boletin de Faculdade de Filosofia, Ciências e Letras da USP n.o 137; Etnografia e tupi-guaranl 23. 1952. v. 1.

7 FIgUeIRA, Luis. Gramátea da lingua do Brasil. Facsimile ed. 1687. Lípsia, J. Platzmann, 1878.

s AYROSA, Plínio. O Caderno de língua ou Vocabulario Portuguez-Tupi de Frel Joăo de Arronches, 1739: notas e comentários a margem de um manuscrpito do sec. XVIII. Revista do Mused Paulista, 21:49-322, 1937.

9 AYROSA, Plinio M.S. Diccionario portuguez-brasiliano e brasiliano-portuguez. Rovista do Musen Paulista, 18:17-322, 1934.

10 AYROSA, Plinio, ed. Vocabulario portuguès-brasilico. Boletim da Faculdade de Fllocofia, Ciência e Letras da USP n. 135; Ełnografia e tupi-guaraní 21, 1951. 113 p. 
embora "não raro aparecem na mesma linha, grafados diversamente: guaçá e goasá..." 10

Não é preciso juntar mais provas; as apresentadas aqui são bastantes para justificar que não se trata de convenção desprovida cie base, mas assenta-se na reprodução relativamente perfeita do tupi elaborada pelos missionários.

A representação gráfica c (ce, ci), ç apoia-se, de modo inconteste, na articulação de um fonema tupi, uma africada sibilante surda, mais ou menos equivalente ao "theta" grego, ao espanhol, graficamente representado por $\mathbf{c}(\mathrm{ce}, \mathrm{ci}) \mathbf{z}$, ou ainda mais ou menos equivalente ao inglês th em vocábulos como third, truth, etc. Ora, sucede que também no português arcaico havia esse fonema, símile ao Co tupi, e fazia-se, portanto, distinção entre c (ce, ci), ç e s (ss). Eram dois fonemas diferentes, e a voga daquele se estendeu até ao decurso do século 16, embora não estejam concordes os estudiosos quanto ao tempo no qual se dera confusão com $s$, e conseqüente desaparecimento da articulação interdental fricativa. ${ }^{11}$ Assim é que se justifica a grafia etimológica de ceio e seio, de cisco e silvar, de maça e massa... E, de acordo com a etimologia, devia-se manter a grafia arcaica çujo, çapato, que, arbitrariamente, são escritos sujo, sapato, pelo princípio estabelecido em todos os sistemas ortográficos que houve em Portugal e no Brasil, de que nenhum vocábulo devia começar por c. Esse princípio tinha a seu favor o desaparecimento da distinção entre ambos os fonemas, e, coerentemente, dever-se-ia dar preferência a s (ss) ou a c (c).

Mas é expressivo que a distinção entre esses fonemas ainda se faz em Portugal, segundo o testemunho de J. Leite de Vasconcelos: "Na raia transmontana, como na do Minho e da Beira e ainda em algumas regiōes do interior, o povo distingue a pronúncia de $\mathbf{s}$ (ss) e c $(c+e, i) \ldots$ Tal distinção de pronúncia observa-se também

11 V. Carolina M. de VASCONCELOS. Liçōes de filosofia portuguesa. Lisboa, Rev. de Portugal 1956 p. 17 e 406: L'a pronúncia de e era ts': fim do séc. 15 ou princípio do 16 (p. 401). "O s é diferenciado do $q$ ao sul do Tejo no século XII" (A.R. GoNCALVES VIANA. Revista Lusitana, $2: 332-8 \ldots$ ) "Já no séc. 13 foi, ao menos ao sul de Portugal, ts simplificou-se em s, conforme se supōe pela escrita de s em rez de c" (J. HUBER. Altportugiesisches Elementarbuch, \$222, nota). Para E.

BOURCIEZ rs $(c+e$, i) do período romano foi reduzido a $s$ em português ai pelo séc. 15. (Eléments de Lingaistique Romane. Paris, Klincksiecè, 1956. p. 406.) A primeira Gramátlea da Lingua portaguesa, de Fernão de OLIVEIRA, faz distinçāo entre c (e, i) e s (3. ed. dir. Rodrigo Sá Nogueira. Lisboa, 1933. p. 35 e 36). Igual distinção faz João de BARROS, cuja segunda gramática lusa, em ordem cronológica, data de 1540 (Gramática da língua portugaesa. 3. ed. org. José Pedro Machado. 1957, p. 62 e 65.) 
na língua antiga de todo o país; funda-se em razões etimológicas... As confusões fonéticas de que falo começaram, termo médio, pelo século XVI". ${ }^{12}$ E o mesmo A. em outra obra: "Sur la frontière d'Entre-Douro-e-Minho, de Trás-os-Montes et de la Beira, et même dans quelques endroits de l'intérieur du pays, l'ancienne distinction se maintient entre c... et s... initiaux et intervocaliques... Dans les autres localités de provinces ci-dessus montionnées, exception faite ce Porto, Lamego et autres villes importantes, $\xi$ et $s$ ont été remplacés respectivament par $s$ (ss)..." ${ }^{13}$

Ora, Anchieta e os demais ao ouvirem, portanto, o som, aqui representado por ts, da língua tupi, registraram-no mediante c, $\mathbf{s}$, c'e acordo com o português da época. De outro modo teriam usado s, ss.

Seria interessante verificar se esse fonema era peculiar ao tupi antigo, ou se, em outras línguas da mesma família, também o possuíam ou se ainda o possuem.

Perlustrando as duas partes da ARTE DE LA LENGUA GUARANI, 0 MAS BIEN TUPI do Pe. Antonio Ruiz de Montoya, 2. ed. 1876, baseada na $1 .^{\circ}$ ed. (TESORO, 1639, e ARTE e VOCABULARIO, 1640) ${ }^{14}$ evidencia-se que os missionários de língua espanhola procederam da mesma maneira. Assim é que nessas obras se nos deparam exemplares destarte grafados: guaçú, açocé, çuçu, teçá, cibá, etc. Igual procedimento tiveram os autores de catecismos, os jesuitas Gaspar de Astete, M. G. de Ripalda, Simón Bandini, Francisco Pomeii, e outros, anônimos, que escreveram em guarani antigo ${ }^{15}$ quaraci, yacitataguaçu, oipiçi, açe, ndeçi, etc.

Por que o mesmo recurso gráfico no guarani por escritores de língua espanhola? Porque no espanhol de outrora, como no da atualidade, existia o mesmo fonema, representado por $c, \boldsymbol{c}$, $\mathbf{x}$. "La lengua antigua, diz M. Pidal, distinguía la ç sorda de la z sonora, cuya pronunciación tuvo que ser originariamente africada o sea una oclusión seguida de una fricativa, que podian representarse por..., cuasi ts, para la c,..." "A comienzos del siglo XVI ya se generalizaba en muchas regiones de la Península la pronunciación interdental, simplemente fricativa...: plaça, hazer. Ambos sonidos se confundieron a

12 LEITE DE VASCONCELLOS, J. Estudos de philologia mirandesa. Lisboa, Imprensa Nacional, 1900. จ.1, p. 34, 35.

12 LEITE DE VASCONCELLOS, J. Esquise d'une dialectologie portagaise. Parls, 1901. p. 114.

14 Nueva ed. Viena, Fresy y Frick, 1876.

15 Boletim da Faculdade de Filosofia, Ciêneias e Letras da USP n. 155, 167, 180, 200 , 212, 213; Etnografia e tupi-guarani 24, 27. 29-32. 1952-56. 6 จ. 
partir del siglo XVII en un solo sordo, perdiéndose el sonoro. Por más que la ortografía moderna distinga la c y la z, las distingue sólo para usar una ante $\mathbf{i}, \mathbf{e}, \mathrm{y}$ otra ante $\mathbf{a}, \mathbf{0} \mathbf{u}$, sin atender a la ortografía antiga etimológica; así que en algunos casos, como sucede en las dos palabras mencionadas, se escriben hoy precisamente al revés de como antes se escribian y pronunciaban: plaza, hacer. Ambas consonantes se pronuncian iguales, con z actual siempre sorda, que acaso es igual a la ç del siglo XVI". ${ }^{10}$ Assim é que, p. ex., Montcya, grafa plaça, hazer, braço, açúcar, etc.

Mas por que Jean de Léry (1578) representou com s, ss tais vocábulos: ouassu, "grande", si, "mãe", assi, "dor", ussuh, "sêde", pussempé, "unha do pé", etc.? 17 Por que Claude d'Abbeville (séc. 17) também grafou com s, ss Camousi, "Camocim", ouassu, "grande", Souassou, "nome de um índio", etc.? ${ }^{(18)}$

Não é difícil explicar o fato; na língua francesa, já no século 13, o som ts, proveniente de $\mathrm{c}+\mathbf{e}$, i latino, reduziu-se a s grafado c: cerf, cité, etc. ${ }^{19}, e$ em conseqüência, veio a concorrer com s- e -ss-: sept, masse, etc.

Aqueles franceses, e outros, não perceberam o som ts, o qual era, para eles, o mesmo s do francês de então, ou se o perceberam, reproduziram-no com o mais aproximado.

Alguns idiomas atuais da família (ou tronco) tupi-guarani conservam ainda esse fonema, comprovando a existência do mesmo no tupi antigo. P. ex., no cocama: tsú, "carne" (tp. çoó), yutsara, "comichão" (tp. iuçara), tayatsú, "porco" (tp. taiaçú), tsúpia, "ovo" (tp. s-upiá), pitsapé, "unha do pé" (tip. pyçapê), mutsa-, "três" (tp. moça-), etc. 20

Em cocamília, ao lado de is (p. ex., motsa-, "três"), há a representação th do inglês this: thái, "dente", etc. ${ }^{21}$

No nheengatu ou tupi amazônico, segundo a análise efetuada,

16 MENENDEZ PIDAL, R. Manual de gramatica historica española. 7. ed. Madrid. 1944. $\$ 35$ bis, nota 2.

17 História de uma viagem feita à terra do Brasil. Trad. de Monteiro Lobato. Rio de Janeiro, 1926. cap. 20 ; LEMOS BARBOSA, A. O diálogo de Léry. Revista Filológłca, (16) :306-22, mar. 1942 .

18 História da missão dos padres capuchinhos na ilha do Maranhåo e terras circunviżnhas. São Paulo, Martins, 1945. 296 p.

19 BOURCIEZ, p. 302 .

20 WILLE, Norma Faust. Vocabulario breve del idioma cocama (tupi). Separata de Peru Indigena, 8:18-9, 1959.

21 TESSMANN, Günter. Die Indianer nordost-Perus. Hamburgo, 1930. p. 66-83. 
por Danielle Granier, Manita Porto e A.D. Rodrigues 22 no disco 7, face 1, do vocabulário do Pe. Alcionílio Brüzzi Alves da Silva ${ }^{23}$, consta uma "fricativa plana interdental surda" (representada aqui por th): thua'thu, "veado" (tp. çuaçú).

No chiriguano de Machareti e no izozó do rio Parapiti ${ }^{24}$ a consoante representada por $s$ é igual a "c en español ante $i$ (como en cielo)", donde dyásy (chir. e iz), "lua" (tp. iacy), yguásu (chir.), "rio grande" (tp. yguaçú), kyse (chir. e iz.), "faca" (tp. kycó), etc.

No guaraju, apanhado por Max Schmidt ${ }^{25}$ o símbolo s é equivalente ao c espanhol em cielo: cheresá, "meu olho", dyasi, "lua", mosapi, "três", etc.

Em um valiosíssimo estudo de fonologia comparada procedido por Wanda Hanke, Morris Swadesh e Aryon D. Rodrigues 26 foi constatada a existência no proto-tupi do fonema fricativo aqui representado por ts, deduzido destes idiomas: tupinambá, kawahib, tembé, aweti, pauserna, sirionó, mawé, mundurucu, xipaya, arikém, mondémayuru e mekéns (e somente conservado neste último): atsu, "grande", -atsi, "māe", etc. E a eles junte-se thap, "casa", thoy, "chuva", bothap-, "panela", da língua cinta-larga (coleta de Sarah Gudschinsky), e dzab, "casa", dzoid, "chuva", bodzab, "panela", dzaga, "matar" (coleta de Harald Schultz) do idioma digüt ${ }^{27}$

Em abono da representação dz, confira-se a coleta de Clive Sandberg ${ }^{28}$, con th, surdo: dath, "cabelo", ta?thu, "burro", ma?thun, "tórax", etc., do cinta-larga.

Em vista do exposto, conclui-se que a transcrição portuguesa do fonema tupi ts (ou th) por c $(+\mathbf{e}, \mathrm{i}), \mathbf{c}(+a, 0, \mathbf{u})$ não foi arbitrária, e, se mais tarde, quando o fonema deixou de ser proferido africado, passando a s, então surgiu a. grafia com s: Sergipe, Iguassu, (hoje Iguaçu, (restabelecida), sabiá ${ }^{29}$, sapucaia, Bossoroca (mas como

Os fonemas do nheengatu, In: REUNIXo BRASILEIRA DE ANTROPOLOGIA, 6. Discotec etnolingüiseico-musieal. Säo Paulo, 1961.

SCHMIDT, Max. Los Chiriguanos e Izozós. Revista de la Socledad Clentifica del Pareguay, v. 4 n. 3, 1938.

Los Guarayú. Revista de la Sociedad Cientifiea del Paraguay, 3(6):176, 1936.

Nota de fonologia Mekens. In: MISCELLANEA Paul Rivet. México, Universidad Nacional Autónoma, 1958. v. 2, p. 187-217. V. também HANKE, Wanda. Breves notas sobre os indios Mondé e o seu idioma. Dusenia, 1(4):215-28, 1950.

7 RODRIGUES, Aryon Dall'Igna. Classificaçāo da língua dos Cinta-Larga. Revista do Antropologis, 14:27-30, 1966.

Cinta Larga. Relatório n. 1 do SIL, 17 abr. 1972.

Em todos os nossos sistemas ortográficos, convencionouse nfo iniciar com $\boldsymbol{s}$ donde sabic, sapucais, etc., e assim também com os de outras origens que nảo tupi: ispato (port. arc. capato), sujo (port. are. cojo), sanefa (are. ganefa). 
substantivo comum boçoroca, voçoroca), Jussara (mas juçara), Urussanga, Pirassununga, Imbassaí, Tupãssi (PR, cp. Tupanci, RS), e mais exemplos poderiam ser aduzidos.

E assim se justifica o restabelecimento, nos últimos sistemas ortográficos do Brasil, do $\mathbf{c}(+\mathbf{e}, \mathbf{i}), \mathbf{f}(+\mathbf{a}, \mathbf{0}, \mathbf{u})$ nos vocábulos de origem tupínica, embora haja exceções.

\section{S U M A R IO :}

O estudo explica por que o fonema (s de vocábulos tupis introduzidos no léxico português não é transcrito com $\mathbf{s}$ (ou ss), mas com $\mathbf{c}$ $(+e, i)$ e ç. $O$ fato se baseia na existência do fonema africano /ts/, no século 16, tanto no tupi quanto no português, neste representado pelas grafias $c(+\mathbf{e}, \mathbf{i})$ e c.

Os primeiros autores portugueses que elaboraram gramáticas, dicionários, etc., da língua tupi, são uniformes nessa representação Jráfica. Igual transcrição foi feita em espanhol pelo Pe. Montoya com relação ao guarani (século 17).

A existência ainda hoje de /ts/ ou de /dz/ em várias línguas da família tupi confirma a asserção, e conclui-se, portanto, que a grafia c, ç em palavras tupis no português não é arbitrária. ceções.

E, por fim, o Autor do estudo explica também por que há ex-

\section{S U M M A R Y :}

The study above explains why the /s/ phoneme from the Tupi words introduced in the Portuguese lexicon is not transcribed into $\mathbf{s}$ (or ss), but into $c(+e, i)$ and $c$. This is due to the existence of the affricate phoneme /ts/ in the sixteenth century both in Tupi and Portuguese, the latter represented by the spelling $\boldsymbol{c}(+\mathbf{e}, \mathbf{i})$ and $c$.

The first Portuguese authors who elaborated the Tupi language grammars, dictionaries, etc. use the same spelling form.

Similar transcription was elaborated in Spanish by Fr. Montoya related to Guarani (17 th century).

Up to now, the existence of $/ \mathrm{t} / \mathrm{s}$ or $/ \mathrm{dz} /$ in several languages belonging to the Tupi family confirms the assertion; therefore, we come to the conclusion that the spelling e, $\mathbf{c}$ in Tupi words in Portuguese is not arbitrary.

At the end, the Author also explains why there are some exceptions. 
1 ABBEville, Claude. História da missāo dos padres eapuchinhos na ilha do Maranhão e terras cirennizinhas. Sāo Paulo, Martins 1945. 296 p.

2 ANCHiETA, José. Arte de gramática da lingua mais usada na costa do Brasil. Ed. facsimilar. São Paulo, Ed. Anchieta, 1946. 58 f.

3 ARAUJo, Antonio. Catecismo na lingas brasilica. Reprod. facsimilar da 1. ed. 1618. Rio de Janeiro, Pontifícia Universidade Católica, 1952. 179 f.

4 AYRosa, Plfnio. O Caderno da língua ou Vocabulário portuguez-tupi de Frei João de Arronches, 1739: notas e comentärios à margem de um manuscrito do séc. XVIII. Revista do Masen Paulista, 21:49-322, 1937.

5 - Diccionario portuguez-brasiliano e brasiliano-portuguez. Revista do Maseu Paulista, 18:17-322, 1934.

6 - Vocabulário português-brasílico: mss. do séc. XVIII. Boletim da Faeuldade de Filosofia, Ciências e Letras da USP n. 137. Etnografia e tupi-guaranı 21, 1951. 113 p.

7 BARBosA. A. Lamos. O Auto de São Lourenço; uma peça teatral de Anchieta em tupi, castelhano e portuguès. Verbum, $7(2): 201-47$, jun. 1940.

8 . O diálogo de Léry. Revista Filológiea, 4(16):306-22, mar. 1942.

9 BOURCIEZ, E. Elements de linguistique romsne. Paris, Klinek sieck, 1956, 783 p.

1) CATECismos vários. Boletim da Faeñdade de Filosofia, Ciêneias e Letras da USP n. 157 167, 180, 200, 212, 213. Etnografia e tupi-guarani 24, 27, 29-32, 1952-56. 6 マ.

11 FIGUEIRA, Luís. Gramática da lingua do Brasil. Facsímile da ed. 1687. Lipsia. J. Platzmann, 1978.

12 GONÇALVES VIANA, A.R. Revista Lusitana n.2, p. 332-8.

13 GRANIER, Danielle et alii. Os fonemas do nheengatu. In: REUNIXO BRASILEIRA DE ANTROPOLOGIA, 6.

14 HANKE, W. Breves notas sobre os indios Mondé e o seu idioma. Dusenim, 1(\$):216-81, 1950 .

15 et alii. Notas de fonologia Mekens. In: MISCELLANEA Paul Riret. México, Universidad Nacional Autônoma, 1958, จ. 2, p. 187-217.

I6 HUBER, J. Altpotagiesisches Elementarbuch.

17 LEITE, Serafim. Leonardo do Vale, autor do primeiro 'Vocabulario na lingua brasilies. Verbum, v. I n. 1, 1944.

18 LEITE DE VASCONCELLOS, J. Esquisse d'une dialectolozie portugaise. Paris, 1901.

I? - Estudos de philologia mirandesa. Lisboa, Imprensa Nacional, 1900.

20 LERY. Jean de. História de uma visgem feita a terra do Brasil. Trad. Monteiro Lobato Rio de Janeiro, 1926.

21 MARTINS, M.L.P. Poesias tupis (séc. XVI). Boletim da Facaldade de Filosofia, Ciêneias e Letras da USP n. 51, 1945.

22 MENENDEZ-PIDAL, R. Manaal de gramatica historica española. 7. ed. Madrid, 1944.

23 OLIVEIRA, Fernảo. Gramática da língua portuguesa. 3. ed. org. José Pedro Maceado. 1957.

24 RODRIGUES, A. Dall'Igna. Classificasão da lingua dos Cinta-Larga. Revista de Antropologia, $14: 27-80,1966$.

25 RUIZ DE MONTOYA, A. Arte de la lengua guarani ó mas bien topi. Nueva ed. Viena, Faesy $y$ Frick, 1876. p. irr.

24 SCHMIDT, Max. Los chriguanos e Izozós. Revista de la Sociedad Cientifica del Paraguay, v. 4, n. 31938.

27 Los Guarayú. Rerista de la Sociedad Cientifica del Paraguay, v. 3 n. 6 , 1936.

23 SILVA, A.B.A. Diseoteca etnolingüistica-musieal. Săo Paulo, 1961.

29 TESSMANN, Günter. Die Indianer nordost-Peras. Hamburgo, 1930.

30 VASCONCELOS. C. Michaelis. Liçōes de filologia portuguesa. Lisboa, Revista do Portugal, 1956, $437 \mathrm{p}$.

31 VOCABULERIO na língua brasilica. 2. ed. rev. por Carlos Drumond. Boletím da Facaldade de Filosofia. Ciências e Letras da USP n. 137. Etnografia e tupi-guaran! 21. 1951. $113 \mathrm{p}$.

32 WILLE, N.F. Vocabulario breve del idioma cocama (tupi). Separata de Pera Indigena n. 8,1959 . 